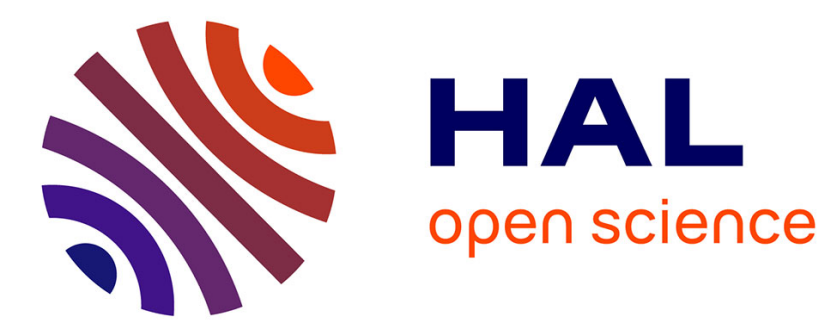

\title{
A Cognitive Vision System for Nuclear Fusion Device Monitoring
}

Vincent Martin, Victor Moncada, Jean-Marcel Travere, Thierry Loarer, François Bremond, Guillaume Charpiat, Monique Thonnat

\section{- To cite this version:}

Vincent Martin, Victor Moncada, Jean-Marcel Travere, Thierry Loarer, François Bremond, et al.. A Cognitive Vision System for Nuclear Fusion Device Monitoring. 8th International Conference, ICVS 2011, Sep 2011, Sophia Antipolis, France. pp.163-172. hal-00609876

\section{HAL Id: hal-00609876 https://hal.science/hal-00609876}

Submitted on 20 Jul 2011

HAL is a multi-disciplinary open access archive for the deposit and dissemination of scientific research documents, whether they are published or not. The documents may come from teaching and research institutions in France or abroad, or from public or private research centers.
L'archive ouverte pluridisciplinaire HAL, est destinée au dépôt et à la diffusion de documents scientifiques de niveau recherche, publiés ou non, émanant des établissements d'enseignement et de recherche français ou étrangers, des laboratoires publics ou privés. 


\title{
A Cognitive Vision System for Nuclear Fusion Device Monitoring
}

\author{
Vincent Martin ${ }^{1}$, Victor Moncada ${ }^{1}$, Jean-Marcel Travere ${ }^{1}$, Thierry Loarer ${ }^{1}$, \\ François Brémond ${ }^{2}$, Guillaume Charpiat ${ }^{2}$, and Monique Thonnat ${ }^{2}$ \\ 1 CEA, IRFM, Bldg. 507, F-13108 Saint Paul Lez Durance, France. \\ 2 INRIA, PULSAR research team, 2004 routes des Lucioles, BP93, F-06902 Sophia \\ Antipolis, France. \\ vincent.martin@cea.fr
}

\begin{abstract}
We propose a cognitive vision-based system for the intelligent monitoring of tokamaks during plasma operation, based on multisensor data analysis and symbolic reasoning. The practical purpose is to detect and characterize in real time abnormal events such as hot spots measured through infrared images of the in-vessel components in order to take adequate decisions. Our system is made intelligent by the use of a priori knowledge of both contextual and perceptual information for ontology-driven event modeling and task-oriented event recognition. The system is made original by combining both physics-based and perceptual information during the recognition process. Real time reasoning is achieved thanks to task-level software optimizations. The framework is generic and can be easily adapted to different fusion device environments. This paper presents the developed system and its achievements on real data of the Tore Supra tokamak imaging system.
\end{abstract}

Keywords: cognitive vision system, infrared monitoring, ontology, multisensor event fusion, thermal event recognition, real-time vision, ITER

\section{Introduction}

Tokamaks are complex devices operated to produce controlled thermonuclear fusion power by magnetic confinement of a plasma (fully ionized gas) in a torus. Even if the temperature drastically decreases from core plasma to edge plasma (from $10^{8}$ to $10^{4}{ }^{\circ} \mathrm{C}$ ), the equilibrium of the plasma discharge requires a direct contact with Plasma Facing Components (PFCs) exposed to very high heat fluxes in the range of 10-15 MW.m ${ }^{-2}$. As a consequence, these PFCs must be continuously monitored to prevent irreversible damages. Infrared (IR) or visible imaging diagnostics (i.e. sensor data analysis system) are routinely used as plasma control systems during operation. The developed systems consist of detecting a high increase of the temperature (i.e. of the IR signal) beyond fixed levels for a set of predefined Regions of Interest (RoIs) [8]. In the perspective of $\mathrm{ITER}^{3}$, with a network equipped with 36 cameras (one mega pixels at $500 \mathrm{~Hz}$ each)

${ }^{3}$ International Thermonuclear Experimental Reactor, first plasma in 2020 
for the IR and visible imaging diagnostic, a complementary approach must be found to alleviate this intensive user-interaction demand. In addition, the future use of metallic components in ITER adds complications in surface temperature estimation of exposed PFCs because of multiple light reflections, making more intricate temperature threshold settings.

Therefore, there exists a real need in improving the performance of such imaging diagnostics. One challenge is to design an intelligent vision system for automatic recognition of thermal/plasma events (also called phenomena) and to embed it into the fusion device environments (see Figure 1 for typical thermal events on two infrared views at Tore Supra). First attempts towards phenomenon recognition for machine protection issues in visible and IR videos are presented in [9] and [7]. Murari et al. [9] propose a bottom-up approach for the automatic recognition of two specific events (plasma instability patterns and dust particles) observed with fast visible and infrared imaging systems during plasma experiments. Martin et al. [7] propose an approach combining prior knowledge of perceptual and contextual information for the automatic recognition of electrical arcing events in infrared videos.

In this paper, we more specifically address the problem of integration of techniques developed so far for global scene understanding with an emphasize on real time system requirements. We propose to use a more advanced image understanding framework based on knowledge driven reasoning. To ensure a high degree of self-adaptability to varying acquisition conditions, we also propose to analyze images without assumptions on absolute temperature measurements. Our framework is inspired from cognitive vision paradigms recently explored [3] in the field of video surveillance applications [12]. More precisely, we propose a system that is reusable for different fusion devices, that can handle unforeseen situations, that can adapt to its environment (i.e. plasma condition awareness), and that can reason from memorized or learned relevant information.

\section{Proposed Approach}

Our cognitive vision system must satisfy three major constraints specific to the fusion device environment. First, it must ensure the maximum security level: a detected but unclassified event remains potentially dangerous (e.g. an unforeseen overheating area), and thus should be handled by the machine protection system. The second constraint refers to the system versatility. Since all fusion devices provide their own diagnostics (i.e. different types of cameras) and several sets of possible phenomena (about 20 per machine), creating all the vision tasks for each environment becomes an intractable task and thus requires to use a shared formalism for knowledge description of both contextual and perceptual information. System performance in term of computational time represents the third major issue since each vision task must fulfill the real time constraint of the considered fusion device environment. Our vision system meets these requirements thanks to the following abilities: 


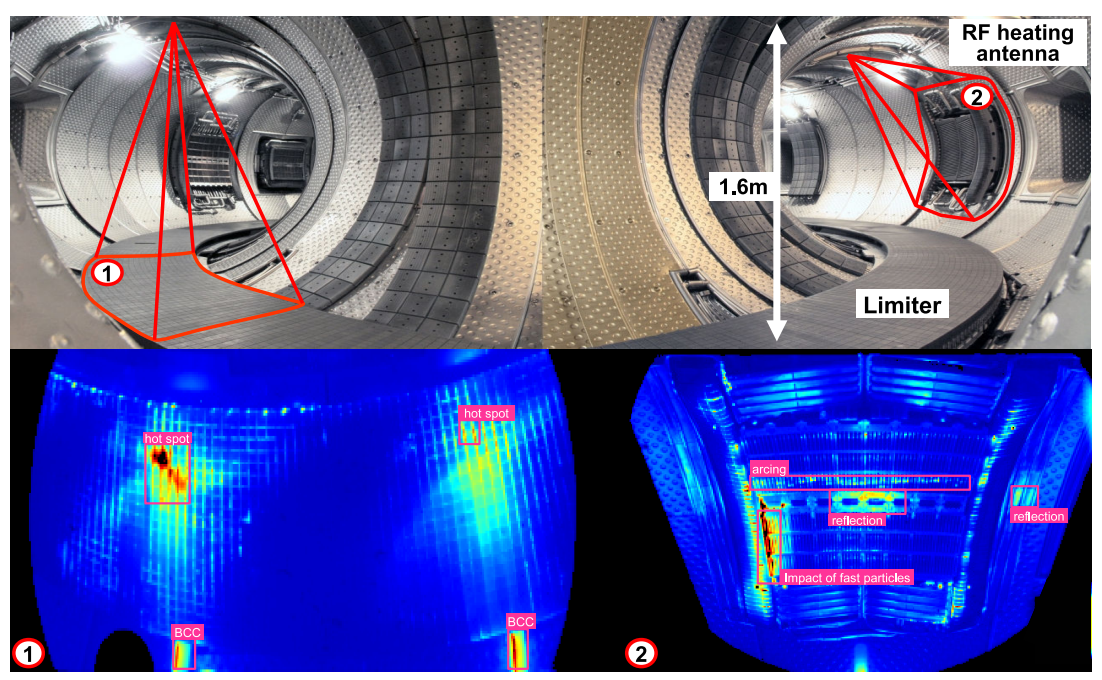

Fig. 1. Infrared monitoring of PFCs in the TS tokamak during a plasma discharge. Temperatures range from $120^{\circ} \mathrm{C}$ (blue) to $900^{\circ} \mathrm{C}$ (dark red). The bounding boxes represent manual annotations defined with the help of physicists. BCC stands for Badly Cooled Component.

Multi-sensor data and event fusion. We merge information at different levels. In a first case, the goal is to find cross-correlation between event features (e.g. similarity between hot spot temperature evolutions) observed at several scene locations. In a second case, an event can generally be detected by several diagnostics (e.g. by IR and visible cameras, by spectroscopy). Combining video events with other sensor events (e.g. a curve peak) helps in achieving high robustness of the system as illustrated in [12].

Ontology-driven vision task composing. The unification of information and especially visual and contextual data can be achieved with ontologies, as demonstrated in [5] and [4]. Our ontology is used to link the different semantic representations related to (1) the diagnostics describing the perceived environment, (2) the scene observed by the camera network, (3) the plasma discharge scenario parameters and (4) the phenomena to be recognized by the system.

Real time vision. To avoid combinatorial explosion of the recognition process based on multi- event hypothesis solving, most of the reasoning is deterministic and performed during the ontology-driven vision task composing by means of spatio-temporal and logic constraint propagation. This approach makes possible task-level optimizations as task pooling and task parallelization mandatory for real time reasoning. Real time constraints imposed by the acquisition systems are also handled thanks to hardware acceleration of low-level algorithms as describe [6]. 


\subsection{Event modeling for vision task composing}

We have developed a software platform dedicated to vision task composing, tuning and launching called PInUP (Plasma Imaging data Understanding Platform). We propose a formal method to infer the vision task hierarchy from prior knowledge described in our ontology. One advantage of this method is to perform two task-level optimizations so as to decrease the CPU loading and thus to save computational time. The first level concerns task pooling used to avoid multiple launching of the same vision algorithm (e.g. detection algorithm). The second level concerns task parallelization which aims at minimizing the length of linear task sequences and is a prerequisite for CPU multi-threading based computing.

The phenomenon ontology branch (see figure 2) contains the class hierarchy of interesting events for machine protection purposes. Each event categorization process is associated with at least one specialized vision task composed of four pipelined algorithms. The specialization of each algorithm is deduced from the attributes of the phenomenon ontology branch. The typical event recognition process consists of:

- A trigger algorithm based on the Plasma scenario attributes such as plasma states. These attributes describe the necessary plasma conditions for the event to occur. This algorithm triggers the launching of the event categorization process.

- A low level detection algorithm deduced from Diagnostic attributes attached to the event. For instance, detection of transient hot spots is performed by a motion detection algorithm.

- A spatial filtering of detected pixels based on the Scene attributes specifying the possible locations of the event. We use an interactive 2D scene model where the user has the possibility to fill symbolic and numerical attributes attached to each defined zone.

- A set of specialized feature descriptors based on the Visual concept attributes (e.g. shape, size, time duration, etc.) selected from prior knowledge of the phenomenon characterization. The corresponding range values are learned from representative training samples.

This ontology-driven construction process leads to the work flow of vision tasks presented in figure 3. Currently, a graphical tool let end-users (e.g. diagnosticians) compose the vision task hierarchy in a user-friendly way by means of component block manipulation.

\section{Application to thermal event recognition at Tore Supra}

The main purpose of thermal event recognition is to handle unforeseen situations as unexpected hot spots. People in charge of the experiments can then focus either on filtered events (e.g. for plasma control issues) or on hot spots remaining unclassified to check their dangerousness. 


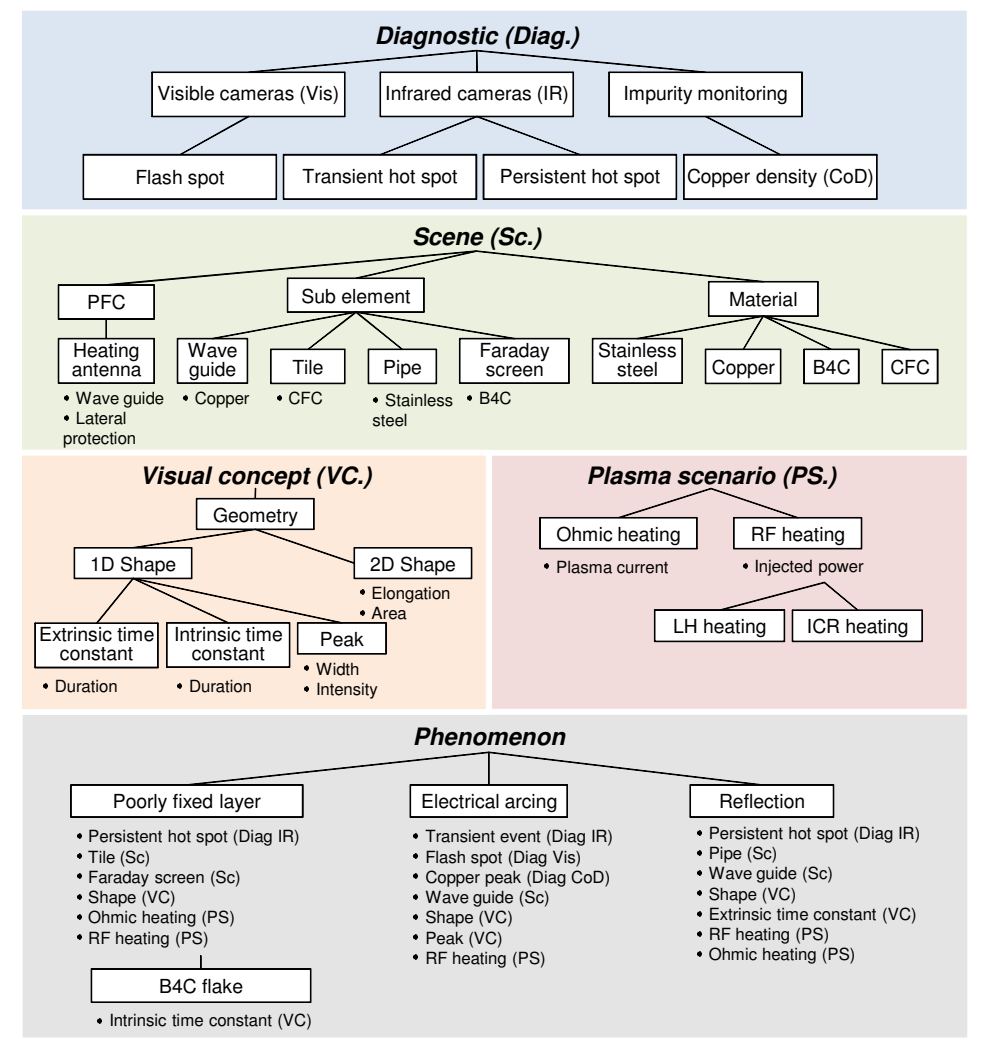

Fig. 2. Part of the ontology developed for thermal event recognition. The five main branches are represented with corresponding class hierarchies and attributes.

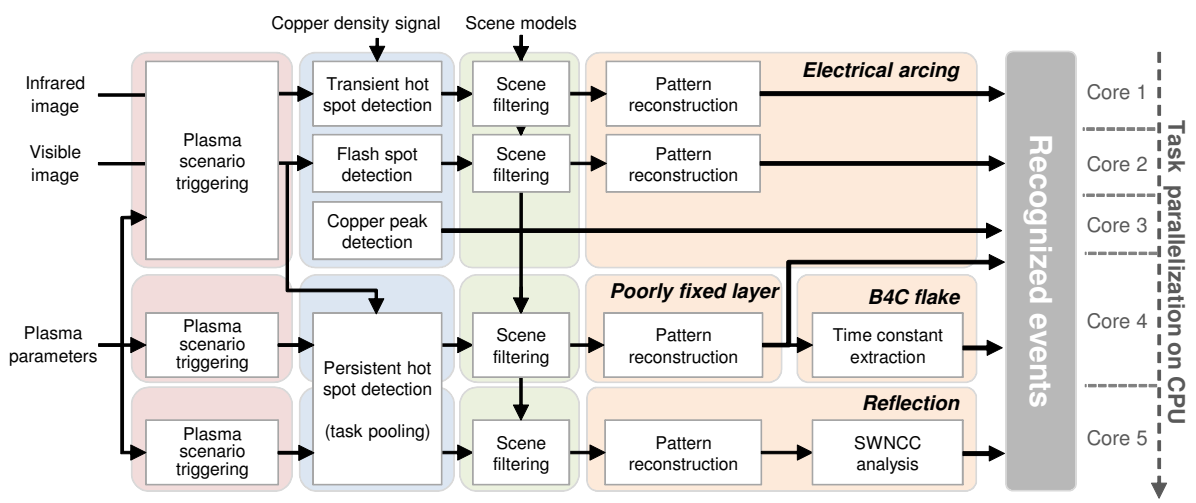

Fig. 3. Work flow of vision tasks for the four phenomena modeled in figure 2 . 


\subsection{Hot spot detection}

Physically, a hot spot is a local area on a PFC where the temperature measured is above an accepted range of values, and is then considered as overheating. Since temperature calibration is still an open problem in tokamak environments, we propose to define the detection of hot spots as a spatial or temporal local image contrast analysis problem, therefore ensuring a high degree of self-adaptability to varying acquisition conditions.

Transient hot spot detection Some events can be characterized thanks to their temporal signature as electrical arcs which last only few dozen of milliseconds. A fast change detector based on pixel intensity is then the best appropriate solution to discriminate them against the other types of hot spots (see figure $4(\mathrm{~b})$ ). To this end, we adopt a pixel-wise background modeling and subtraction technique developed by Butler et al. [2].

Persistent hot spot detection All hot spots lasting more than few dozen of milliseconds are considered as persistent. Since time is not necessary a discriminant clue for these hot spots, we adopt a local adaptive thresholding technique for their detection. After extensive tests of state-of-the-art algorithms [10], we found that the efficient implementation of the Sauvola's method based on integral images [11] gives the best results on our data (see figure 4(c)). Using some improvements for computational purposes, the thresholded image $q(x, y)$ from an input image $I$ with pixel intensities $p(x, y)$ is such that:

$$
\forall(x, y) \in I, \quad q(x, y)= \begin{cases}0 & \text { if } p(x, y)<\tau(x, y) \quad \text { or } \quad p(x, y) \leq R \\ 255 & \text { otherwise }\end{cases}
$$

where

$$
\tau(x, y)=\mu(x, y)+k\left(\mu(x, y)-\max _{I}(p)\right)\left(\frac{2 \sigma(x, y)}{\max _{I}(p)-\min _{I}(p)}-1\right)
$$

with $\mu(\cdot)$ and $\sigma(\cdot)$ are the average and standard deviation values of pixel intensities in the spatial neighbourhood centered on $(x, y)$, and $R=\arg \max \{h i s t(I)\}$.

We have also extended the algorithm to adaptive multi-threshold image segmentation through a $n$-pass procedure in order to better discriminate very hot spots inside hot regions as seen in figure $4(\mathrm{c})$.

\subsection{Hot spot categorization}

The next steps after detecting hot spots consist in extracting semantic information based on their appearance and behaviour. In the scope of machine protection issues, this step is of primary importance to assess hot spot dangerousness. 


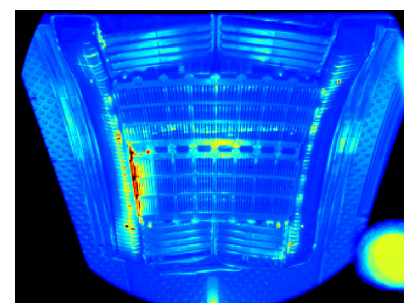

(a)

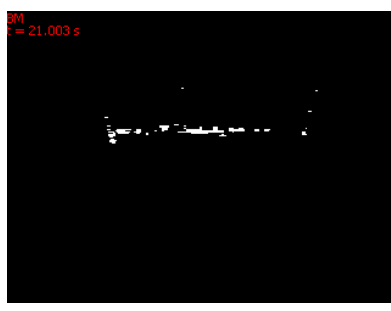

(b)

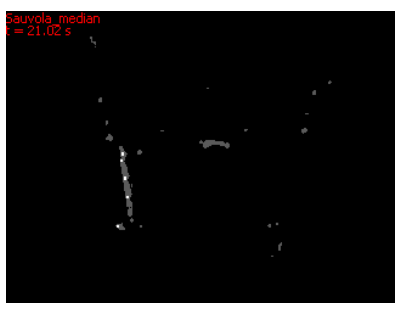

(c)

Fig. 4. Hot spot detection results on an infrared image of Ts heating antenna (a). Transient hot spots (b) and persistent hot spots (c) are clearly discriminated thanks to the two dedicated algorithms.

Persistent Hot Spot Categorization. Among all the modeled thermal events, one have to pay attention to the reflection event. Indeed, reflections patterns correspond to false hot spots which are not per se dangerous. They can be observed on reflective materials e.g. copper, stainless steel but generally not carbon considered closed to a black body behaviour. Reflections appear when PFCs in direct contact with the plasma experience surface temperature in the range of $1000-2000^{\circ}$ C. At Tore Supra, reflections arise from hot regions mainly located on the device floor called the limiter. A direct approach to match a reflection source and a reflection pattern is to measure the similarity between the temporal evolutions of their temperature. To this end, we compute the normalized crosscorrelations (NCC) on a sliding time window (SWNCC) of width $T$ between candidates of both sources $f$ (hot spots on the limiter) and reflection patterns $g$ (hot spots on metal-made PFCs) using the maximal temperature of each hot spot as input feature:

$$
\mathrm{SWNCC}=\frac{1}{T} \sum_{u=t-T}^{t} \frac{(f(u)-\bar{f})(g(u)-\bar{g})}{\sigma_{f} \sigma_{g}}
$$

with $f$ and $g$ the maximal temperatures of the detected hot spots and $\bar{f}, \bar{g}, \sigma_{f}, \sigma_{g}$ their respective averages and standard deviations over the time period $T$.

Figure 5 presents the result of multi-camera event feature fusion performed on two synchronized infrared cameras. The goal is to characterize reflection patterns on the monitored heating antenna while applying radio-frequency (RF) plasma heating. As seen in figure 5, the temperature evolution of three patterns (no. 32, 38 and 45) are well-correlated (SWNCC $>0.9$ ) with the hot spot pattern detected on the limiter (no. 1) and then can be classified as reflection patterns. On the contrary, the pattern no. 27 has clearly an independent thermal behaviour, and will remain classified as persistent hot spot for further analysis and event classification refinement.

Transient Hot Spot Categorization. We present in figure 6 the result of arc recognition with infrared and visible imaging diagnostics during a same plasma discharge. 

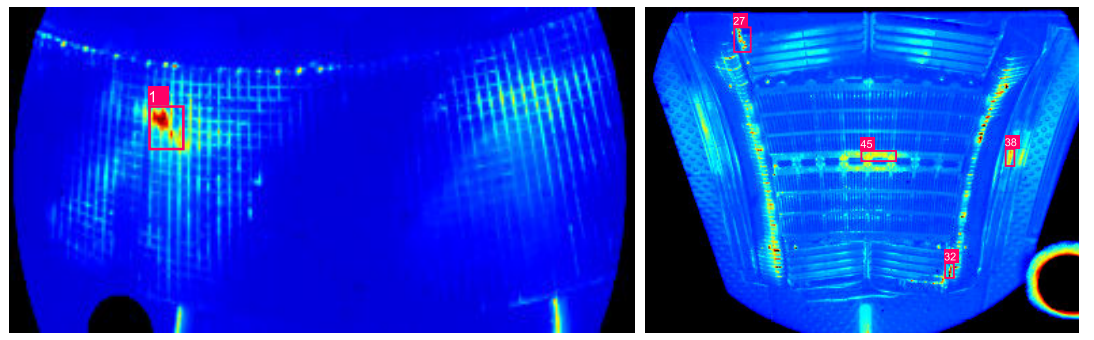

(a) Hot spot detected on the limiter and (b) Candidates of reflection used as heat source for the computation of patterns used for the compuSWNCC. tation of SWNCC.

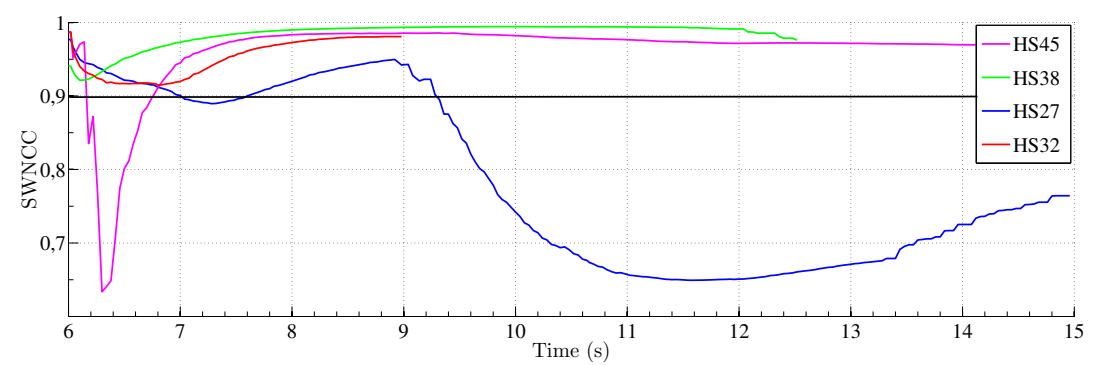

(c) Results of SWNCC $(T=10 \mathrm{~s})$ between the temperature evolutions of the hot spot in (a) and hot spots in (b) during a plasma discharge. Hot spots detected on metallic components of the heating antenna with a SWNCC $>0.9$ are considered as reflection patterns.

Fig. 5. Example of multi-camera event feature fusion for the recognition of reflection patterns based on thermal behaviour cross-correlation.
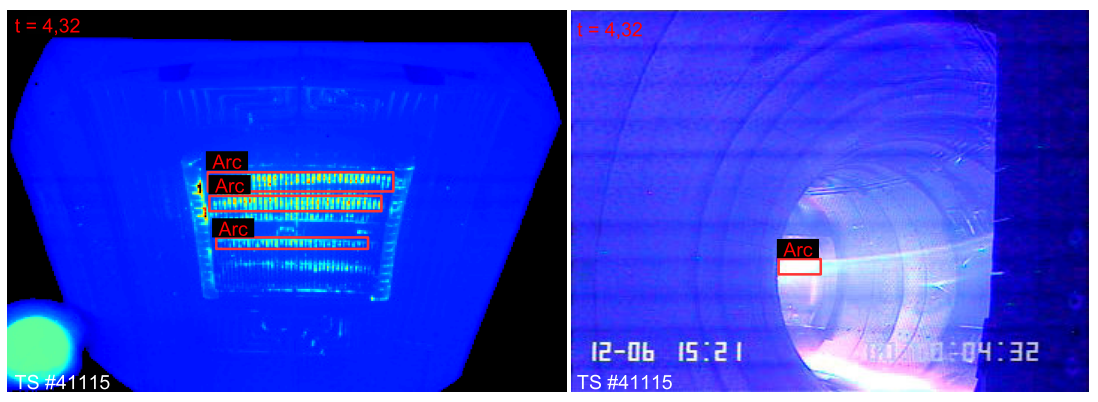

Fig. 6. Synchronized IR (left) and visible (right) camera views aiming the same PFC (heating antenna) from different lines of sight inside the TS tokamak. The arc event is successfully recognized by the system in the two cases. 
Although both sensors data and observed scenes are completely different, the system achieves the recognition of the same arc event appearing in front of the same heating antenna. This ability of correlating events recognized with several diagnostics is considered as an essential element for plasma control system reliability in case of unexpected failure of one diagnostic during plasma operation.

\section{Validation of Experimental Results}

Our vision system is currently implemented on the TS tokamak in parallel of the existing RoI-based monitoring system. The infrared viewing system is composed of 7 infrared video cameras $(320 \times 240$ pixels at $50 \mathrm{~Hz})$ monitoring one part of the toroidal limiter and the five RF heating antennas as seen on top of figure 1 (resp. (1) and (2)). Our system has been tested and validated with plasma physicists during the last experimental campaign on about 50 plasma discharges lasting between 15 and 90 seconds. Performance of arc recognition based on the presented framework has been evaluated in [7] thanks to existing ground truth data (visual annotations). Results shows a precision of $98 \%$ and a sensitivity of $92 \%$. The validation of the SWNCC method used for the characterization of reflection patterns is based on a qualitative comparison with simulated infrared images obtained with a realistic photonic simulation code described in [1]. Results shows that recognized reflection patterns are in accordance in terms of location and size with those found in simulated images. Further quantitative evaluation using this new simulation tool are foreseen to assess the effectiveness of the SWNCC method for reflection pattern recognition in different contexts (i.e. various plasma conditions and monitored PFCs).

\section{Conclusion}

In this paper, we demonstrate that a cognitive vision system based on qualitative imaging analysis can achieve a physical interpretation of observed phenomena during plasma operation in tokamaks. From a computer vision viewpoint, this real system is made original by the merging of multiple sources of information (multi-camera and multi-sensor data fusion) at different levels (pixels and event features) during the recognition task, its combination of different software optimization schemes for real time computations, and the use of ontology-driven inference mechanisms for system re-usability on different tokamak environments. The developed software platform is now daily used at Tore Supra during plasma operation as a computer-aided decision support system and is going to be installed on $\mathrm{JET}^{4}$, which is currently the world's biggest tokamak. On-going work concerns the system performance evaluation to prepare its integration within the plasma control system of Tore Supra.

Finally, this pioneer work for the domain is also an opportunity for both computer science and plasma science communities to progress together for preparing both the safety and physics exploitation of ITER.

\footnotetext{
${ }^{4}$ Joint European Torus
} 


\section{Acknowledgment}

This work was supported in part by the European Communities under the contract of Association between EURATOM, CEA and the French Research Federation for fusion studies. The views and opinions expressed herein do not necessarily reflect those of the European Commission. This work was also supported by the French Stimulus Plan 2009-2010.

\section{References}

1. Aumeunier, M.H., Travere, J.M., Loarer, T., Benoit, F.: Simulation vs. reality: a step towards a better understanding of infrared images in fusion devices. IEEE Transactions on Plasma Science (August 2011), to appear

2. Butler, D., Bove, Jr., V.M., Sridharan, S.: Real-time adaptive foreground/background segmentation. EURASIP J. Appl. Signal Process. 2005, 2292$2304(2005)$

3. Eggert, J., Wersing, H.: Approaches and challenges for cognitive vision systems. In: Sendhoff, B., Krner, E., Sporns, O., Ritter, H., Doya, K. (eds.) Creating Brain-Like Intelligence, Lecture Notes in Computer Science, vol. 5436, pp. 215-247. Springer Berlin / Heidelberg (2009)

4. Gomez-Romero, J., Patricio, M., Garcia, J., Molina, J.: Ontological representation of context knowledge for visual data fusion. In: Int. Conf. on Information Fusion. pp. $2136-2143(2009)$

5. Maillot, N., Thonnat, M., Boucher, A.: Towards ontology-based cognitive vision. Machine Vision and Applications 16, 33-40 (2004)

6. Martin, V., Dunand, G., Moncada, V., Jouve, M., Travere, J.: New FPGA-based image-oriented acquisition and real-time processing applied to plasma facing component thermal monitoring. Rev. of Sci. Instr. 81(10), 10E113 -10E113-4 (oct 2010)

7. Martin, V., Travere, J., Brémond, F., Moncada, V., Dunand, G.: Thermal event recognition applied to protection of tokamak plasma-facing components. IEEE Trans. on Instr. and Meas. 59(5), 1182-1191 (2010)

8. Moreau, P., Barana, O., Brémond, S., Colas, L., Ekedahl, A., Saint-Laurent, F., Balorin, C., Caulier, G., Desgranges, C., Guilhem, D., Jouve, M., Kazarian, F., Lombard, G., Millon, L., Mitteau, R., Mollard, P., Roche, H., Travere, J.: RF heating optimization on Tore Supra using feedback control of infrared measurements. Fusion Engineering and Design 82(5-14), 1030-1035 (2007)

9. Murari, A., Camplani, M., Cannas, B., Mazon, D., Delaunay, F., Usai, P., Delmond, J.: Algorithms for the automatic identification of MARFEs and UFOs in JET database of visible camera videos. IEEE Trans. on Plasma Science 38(12), 3409-3418 (2010)

10. Sezgin, M., Sankur, B.: Survey over image thresholding techniques and quantitative performance evaluation. Journal of Electronic Imaging 13(1), 146-168 (2004)

11. Shafait, F., Keysers, D., Breuel, T.M.: Efficient implementation of local adaptive thresholding techniques using integral images. In: SPIE Conference Series. vol. 6815 (Jan 2008)

12. Zouba, N., Brémond, F., Thonnat, M.: Multisensor fusion for monitoring elderly activities at home. In: Proceedings of AVSS '09. pp. 98-103. IEEE Computer Society, Washington, DC, USA (2009) 